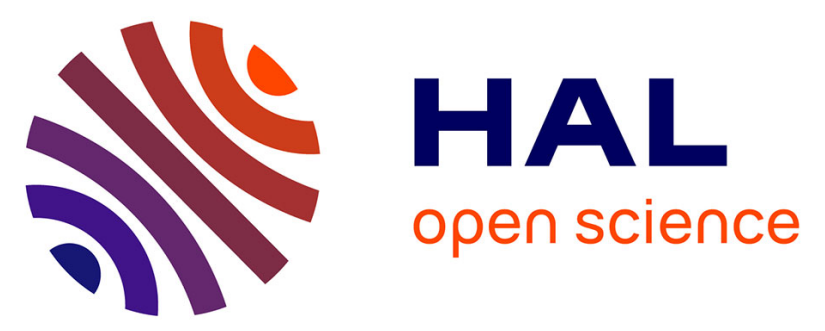

\title{
Geometrical Method Using Simplicial Cones for Overdetermined Nonnegative Blind Source Separation: Application to Real PET Images
}

Wendyam S. B. Ouedraogo, Antoine Souloumiac, Mériem Jaidane, Christian Jutten

\section{To cite this version:}

Wendyam S. B. Ouedraogo, Antoine Souloumiac, Mériem Jaidane, Christian Jutten. Geometrical Method Using Simplicial Cones for Overdetermined Nonnegative Blind Source Separation: Application to Real PET Images. LVA/ICA 2012 - 10th International Conference on Latent Variable Analysis and Signal Separation, Mar 2012, Tel-Aviv, Israel. pp.494-501. hal-00687436

\section{HAL Id: hal-00687436 https://hal.science/hal-00687436}

Submitted on 13 Apr 2012

HAL is a multi-disciplinary open access archive for the deposit and dissemination of scientific research documents, whether they are published or not. The documents may come from teaching and research institutions in France or abroad, or from public or private research centers.
L'archive ouverte pluridisciplinaire HAL, est destinée au dépôt et à la diffusion de documents scientifiques de niveau recherche, publiés ou non, émanant des établissements d'enseignement et de recherche français ou étrangers, des laboratoires publics ou privés. 


\title{
Geometrical Method Using Simplicial Cones for Overdetermined Nonnegative Blind Source Separation: Application to Real PET Images
}

\author{
Wendyam S.B. Ouedraogo ${ }^{1,2,3}$, Antoine Souloumiac ${ }^{1}$, \\ Meriem Jaidane ${ }^{2}$, and Christian Jutten ${ }^{3}$ \\ 1 CEA, LIST, Laboratoire d'Outils pour l'Analyse de Données, \\ Gif-sur-Yvette, F-91191, France \\ 2 Unité Signaux et Systèmes, École Nationale d'Ingénieurs de Tunis, \\ BP 37, 1002 Tunis, Tunisie \\ 3 GIPSA-lab, UMR 5216 CNRS, Université de Grenoble, 961 rue de la Houille \\ Blanche BP 46 F-38402 Grenoble Cedex, France \\ wendyam-serge-boris.ouedraogo@cea.fr
}

\begin{abstract}
This paper presents a geometrical method for solving the overdetermined Nonnegative Blind Source Separation (N-BSS) problem. Considering each column of the mixed data as a point in the data space, we develop a Simplicial Cone Shrinking Algorithm for Unmixing Nonnegative Sources (SCSA-UNS). The proposed method estimates the mixing matrix and the sources by fitting a simplicial cone to the scatter plot of the mixed data. It requires weak assumption on the sources distribution, in particular the independence of the different sources is not necessary. Simulations on synthetic data show that SCSA-UNS outperforms other existing geometrical methods in noiseless case. Experiment on real Dynamic Positon Emission Tomography (PET) images illustrates the efficiency of the proposed method.
\end{abstract}

Keywords: Blind Source Separation, Nonnegativity, Simplicial Cone, Minimum Volume, Facet.

\section{Introduction}

We deal with the problem of Nonnegative Blind Source Separation (N-BSS) in noiseless, linear intantaneous mixture case. The mixture model is given by:

$$
\underset{m \times p}{X}=\underset{m \times n n \times p}{A} \underset{m \times}{S}
$$

where $m$ is the number of observations, $n$ is the number of sources and $p$ is the number of samples. $X, A$ and $S$ are respectively the given observations matrix, the unknown mixing matrix and the hidden nonnegative sources matrix. N-BSS consists on retrieving $S$ and $A$ given only $X$. Possible directions for solving problem (1) are the geometrical approaches. These methods are very natural and intuitive and require weak assumption on the sources distribution. The first geometrical method 
was introduced by Puntonet et al. 1] for separating two sources having bounded probability density functions. The mixing matrix is retrieved by finding the slopes of the parallelogram containing the scatter plot of mixed data. Babaie-Zadeh et al. 2] propose another geometrical method applicable to more than two sources based on clustering the observations points and fitting a line (hyper-plane) to each cluster to recover the mixing matrix. By assuming the (very strong) condition local dominance of the sources (i.e. for every source there is at least one instant where the underlined source is active and all the others are not), several authors propose estimating the mixing matrix by looking for the vertices of the convex hull of the scatter plot of mixed data 3$][4$. These methods can unfortunately be very slow and demanding very large size samples, specially for large scale problem due to the convex hull computing. Noting that when the sources are nonnegative, the scatter plot of mixed data is contained in the simplicial cone generated by the mixing matrix, other geometrical methods were proposed for solving problem (1) by looking for the Minimum Volume (MV) simplicial cone containing the mixed data [5] 6]. MV like methods do not require local dominance of sources. But the simplicial cone generated by the mixing matrix must be well recognizable from the scatter plot of mixed data. This weaker condition implies that there should be at least $n-1$ mixed data points on, or close to, each facet of this cone. It's also necessary to specify that beside the nonnegativity, some MV like methods developed for hyperspectral data processing [5] 6] require full additivity of the sources (i.e. the sum on every column of the sources matrix is equal to one).

This paper presents a MV like method for solving overdetermined N-BSS problem called Simplicial Cone Shrinking Algorithm for Unmixing Nonnegative Sources (SCSA-UNS). This work establishes an extension of [9] in which we only consider the determined case with nonnegative mixing matrix. Section 2 reviews the geometrical view of the N-BSS problem and derives the main idea of MV like methods. In section 3, we describe the proposed SCSA-UNS method. Section 4 presents simulation results on synthetic data and real Dynamic Positon Emission Tomography (PET) data and comparisons with other MV like methods. Finally section 5 presents the conclusions and future works.

\section{Geometrical View of N-BSS Problem}

Let's review the geometrical view of the N-BSS problem we described in [9]. For a given matrix $W=\left[w_{1}, w_{2}, \cdots, w_{n}\right]$, we define the Simplicial Cone $\operatorname{Span}^{+}(W)$ generated by $W$ by :

$$
\operatorname{Span}^{+}(W)=\left\{z \mid z=W y \text { with } y \in \mathbb{R}_{+}^{n}\right\}
$$

We also define the positive orthant $\mathbb{R}_{+}^{n}$ as being the simplicial cone generated by the identity matrix $I_{n}: \mathbb{R}_{+}^{n}=\operatorname{Span}^{+}\left(I_{n}\right)$

By considering each column $x_{i}$ of $X(1 \leq i \leq p)$ as a point in the $n$ dimension data space, it comes that when the sources are nonnegative, the mixed data form a cloud of points contained in the simplicial cone generated by the mixing matrix.

$$
\left\{x_{i} \mid x_{i} \in X, 1 \leq i \leq p\right\} \subseteq \operatorname{Span}^{+}(A)
$$


One can thus imagine to estimate $A$ (up to the classical (positive here) scaling and permutation BSS indetermination's) by finding a simplicial cone containing all the mixed data. But without any additional condition there is an infinite number of such cones. So for recovering the mixing matrix, we require the scatter plot of the mixed data to fill enough $\operatorname{Span}^{+}(A)$ and we look for the Minimum Volume (i.e. the smallest) simplicial cone containing the mixed data [7. Filling enough means that $\operatorname{Span}^{+}(A)$ must be well recognizable from $\left\{x_{i}, x_{i} \in X, 1 \leq i \leq p\right\}$ (i.e. there should be at least $n-1$ mixed data points on, or close to, each facet of $\operatorname{Span}^{+}(A)$ [8]). This intuitive and natural condition will be defined more formally in a future work.

\section{Geometrical Method Using Simplicial Cones for Overdetermined Nonnegative Blind Source Separation}

\subsection{Determined Case : Simplicial Cone Shrinking Algorithm for Unmixing Nonnegative Sources (SCSA-UNS)}

We first restrict to determined case with full column rank nonnegative mixing matrix (i.e. $m=n$ and $A \geq 0$ ). This case is considered in [9]. SCSA-UNS aims at estimating $A$ by finding the Minimum Volume simplicial cone containing all the mixed data. In this objective, we propose a criterion for measuring the volume of a given simplicial cone and an algorithm to minimize this criterion.

Proposed Criterion : We define $V(W)$, the volume of a simplicial cone $\operatorname{Span}^{+}(W)$ generated by a given square matrix $W=\left[w_{1}, w_{2}, \cdots, w_{n}\right]$, where $w_{i}$ is the $i$-th column of $W$, by :

$$
V(W)=\frac{|\operatorname{det}(W)|}{\left\|w_{1}\right\| \times\left\|w_{2}\right\| \times \cdots \times\left\|w_{n}\right\|}
$$

$V(W)$ strictly represents the "aperture" of the simplicial cone $\operatorname{Span}^{+}(W)$, it is positive and upper bounded by 1 (Hadamard's Inequality).

The task of estimating the mixing matrix can then be reduced to solving the following optimization problem:

$$
W^{*}=\underset{W \geq 0, W^{-1} X \geq 0}{\arg \min } V(W)
$$

Proposed Algorithm : We define the $R_{k}^{l}$ like matrices by :

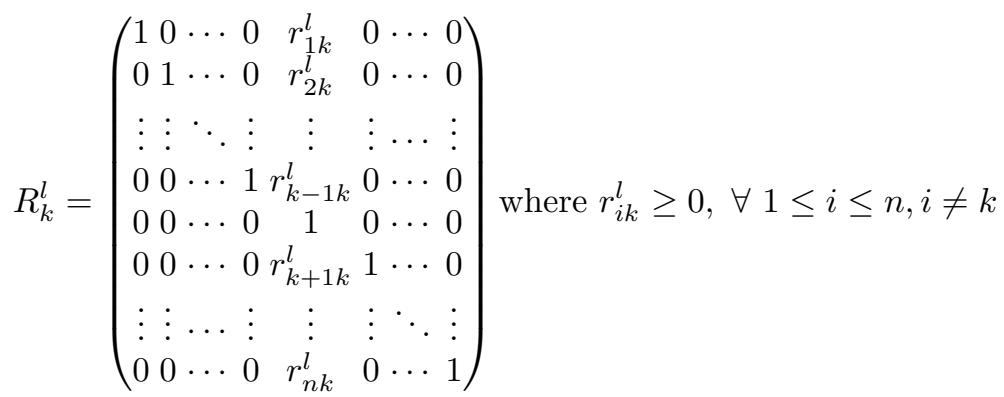


Proposition 1. For a given nonnegative matrix $W$, the volume $V(W)$ of $\operatorname{Span}^{+}(W)$ decreases when $W$ is multiplied to the right by $R_{k}^{l}, \forall l$ and $\forall 1 \leq k \leq n$.

Proof. For fixed $l$, fixed $k(1 \leq k \leq n)$ and a given nonnegative square matrix $W=\left[w_{1}, w_{2}, \cdots, w_{n}\right]$, let $U=W R_{k}^{l}\left(U=\left[u_{1}, u_{2}, \cdots, u_{n}\right]\right)$. The task is to demonstrate that $V(U) \leq V(W)$ :

1. $|\operatorname{det}(U)|=\left|\operatorname{det}\left(W R_{k}^{l}\right)\right|=|\operatorname{det}(W)|\left|\operatorname{det}\left(R_{k}^{l}\right)\right|=|\operatorname{det}(W)| \operatorname{because} \operatorname{det}\left(R_{k}^{l}\right)=1$ 2. $\forall j \neq k, u_{j}=w_{j} \Longrightarrow\left\|u_{j}\right\|=\left\|w_{j}\right\|$

3. For $j=k, \forall 1 \leq i \leq n, u_{i k}=w_{i k}+\sum_{q=1, q \neq k}^{n} w_{i q} r_{q k}^{l} \geq w_{i k}$ because $w_{i q}$ and $r_{q k}^{l}$ are all nonnegative. Therefore $\left(u_{i k}\right)^{2} \geq\left(w_{i k}\right)^{2}$ which leads to $\left\|u_{k}\right\| \geq\left\|w_{k}\right\|$ According to the definition of the volume of a simplicial cone given by (4), 1 ., 2. and 3. allow us to conclude that $V(U) \leq V(W)$

For solving problem (5), SCSA-UNS starts from an initial simplicial cone containing all the mixed data (typically, the positive orthant $\operatorname{Span}^{+}\left(I_{n}\right)$ ) and iteratively decreases its volume by performing several sweeps of $n$ right-multiplications of the matrix which generated this initial cone $\left(W_{0}=I_{n}\right)$ by the $R_{k}^{l}$ matrices $(1 \leq k \leq n)$. At each iteration, the matrix $R_{k}^{l}$ is computed so to keep the mixed data inside the new simplicial cone. Details for computing the $R_{k}^{l}$ are given in Appendix 1 . Let $W \geq 0$ be the matrix which generated the current simplicial cone ( $W$ is the current estimation of the mixing matrix and $Y=W^{-1} X \geq 0$ is the current estimation of the sources). The algorithm stops when one cannot decrease anymore $V(W)$ without creating negative values in $Y$. This often corresponds to the convergence of $W$ to the true mixing matrix $A$. However, it may happen that $V(W)$ does not decrease anymore during the iterations while $W$ has not converged yet to $A$. This freezing 1 situation arises when there is at least one zero value on each row of $Y$. To avoid this problem, we suggest applying, after each sweep $l$, an orthogonal linear transformation $Q_{l}$ to $Y$ (and $Q_{l}^{T}$ to $W$ ) to delete the zeros values of $Y$ without increasing $V(W)$. The details of computing the unfreezing matrices $Q_{l}$ are given in Appendix 2 .

The whole estimated mixing matrix and sources are given by:

$$
A=W_{0} \prod_{l}\left[\left(\prod_{k=1}^{n} R_{k}^{l}\right) Q_{l}^{T}\right] \quad \text { and } S=A^{-1} X
$$

\subsection{Proposed Method for Overdetermined Case}

When the number of observations is greater than the number of sources $(m>$ $n$ ), we propose to first perform a dimension reduction of the mixed data and afterward run the SCSA-UNS algorithm on the reduced mixed data.

Dimension Reduction : The dimension reduction is performed by the classical Principle Component Analysis (PCA). We compute the Singular Value Decomposition (SVD) of the mixed data and we keep only the $n$ largest singular values and the corresponding singular vectors.

\footnotetext{
${ }^{1}$ See Appendix 2 for more details.
} 


$$
\underset{m \times p}{X} \approx \underset{m \times n n \times n n \times p}{E} \underset{G^{T}}{F} G^{T}
$$

The SCSA-UNS algorithm should be executed on $G^{T}$ but $G^{T}$ is not necessarily nonnegative, so its scatter plot is not necessarily contained in the positive orthant. One must then find an initial simplicial cone containing all the dimension reduced mixed data $G^{T}$, since $\operatorname{Span}^{+}\left(I_{n}\right)$ is not necessarily suited any more.

Widening Procedure for Initialization : If all the observations are not nonnegative, we have developed a procedure for finding a convenient initial simplicial cone containing all the mixed for the SCSA-UNS algorithm. This procedure is based on widening $\operatorname{Span}^{+}\left(I_{n}\right)$ by multiplying $I_{n}$ by $D_{k}^{l}$ matrices, with a structure similar to $R_{k}^{l}$, but with negative entries in order to increase the volume of $\operatorname{Span}^{+}\left(I_{n}\right)$ up to enclose all the mixed data. The details of computation of the $D_{k}^{l}$ are not given here due to lack of space. This widening procedure is used on $G^{T}$ to compute $W_{1}$ and $Y_{1}$ so that:

$$
\underset{n \times p}{G^{T}}=\underset{n \times n n \times p}{W_{1}} Y_{1} \quad \text { with } \quad Y_{1} \geq 0
$$

Estimating the Mixing Matrix and the Sources : The SCSA-UNS algorithm is finally executed on $Y_{1}$ to give $\underset{n \times p}{Y_{1}}=\underset{n \times n n \times p}{W_{2}} Y_{2}$.

The whole mixing matrix and sources are estimated by:

$$
\underset{m \times n}{A} \approx \underset{m \times n n \times n_{n \times n n \times n}}{E} W_{1} W_{2} \text { and } \underset{n \times p}{S} \approx \underset{n \times p}{Y_{2}}
$$

\section{Simulations and Discussions}

\section{Case 1: Synthetic Data}

The proposed method is first evaluated on synthetic data and compared with two other MV methods: MVSA [5] and MVES [6]. To make sure that the mixed data fill enough the simplicial cone generated by the mixing matrix, the nonnegative sources have been generated using "random sparse uniform distribution generator" with $64 \%$ of non-zero elements in the sources matrix. We consider two cases: full additive sources and non full additive sources. The mixing matrix has Gaussian random entries. We set $m=20, n=5$ and $p=10000$. Comparison criteria are the CPU time to converge $T$ and the separation error $E_{\text {sep }}$ defined by (11). The smaller $E_{\text {sep }}$, the better the separation.

$E_{\text {sep }}=\frac{1}{n(n-1)}\left[\sum_{i}\left(\sum_{j} \frac{\left|\left(W^{-1} A\right)_{i j}\right|}{\max _{k}\left|\left(W^{-1} A\right)_{i k}\right|}-1\right)+\sum_{j}\left(\sum_{i} \frac{\left|\left(W^{-1} A\right)_{i j}\right|}{\max _{k}\left|\left(W^{-1} A\right)_{k j}\right|}-1\right)\right]$

Table 1 records the average performance indices for 50 independent MonteCarlo runs. One may note that when the sources are full additive, the three methods perform a good separation but SCSA-UNS is faster than MVSA and MVES. However when the sources are not full additive, SCSA-UNS still performs a perfect separation while MVSA and MVES do not. 
Table 1. Average performance indices

\begin{tabular}{|l|c|c|c|r|}
\hline & Index & SCSA-UNS & MVSA & MVES \\
\hline Full additive sources & $E_{s e p}$ & $3,56.10^{-10}$ & $1,23.10^{-12}$ & $2,67.10^{-8}$ \\
& $T(s)$ & 0.69 & 2,21 & 8,37 \\
\hline Non full additive sources & $E_{s e p}$ & $1,54.10^{-9}$ & 7,89 & 6,01 \\
& $T(s)$ & 0,83 & 4,76 & 5,59 \\
\hline
\end{tabular}

Case 2: Real Dynamic Positon Emission Tomography (PET) Images Simulations have also been performed on real Dynamic Positon Emission Tomography (PET) data to study the pharmacokinetics of the [18F]-FDG (FluoroDeoxyGlucose) tracer on human brain. The main objective is to estimate the arterial pharmacokinetic also called Arterial Input Function (AIF) using only the dynamic TEP images with no arterial blood sampling (rAIF) which is too invasive for routine clinic use. The rAIF is only considered here as the reference AIF to assess the proposed estimator accuracy. As a matter of fact, an accurate estimation of the AIF allows a quantitative measurement which is indispensable for an efficient treatment evaluation in oncology. We have 19 human brain PET images recorded during $33 \mathrm{mn}$. Each $3 \mathrm{D}$ PET image is reshaped to form one row of the observations matrix $X$. The number of observations is $m=19$ and the number of samples is $p=266742$. We set the number of sources to $n=4$.

Fig 1 shows the pharmacokinetics compartments estimated by the SCSA-UNS algorithm. Every subfigure represents the normalized kinetics (estimated mixing matrix) over the first four minutes (lower left) and the corresponding spatial
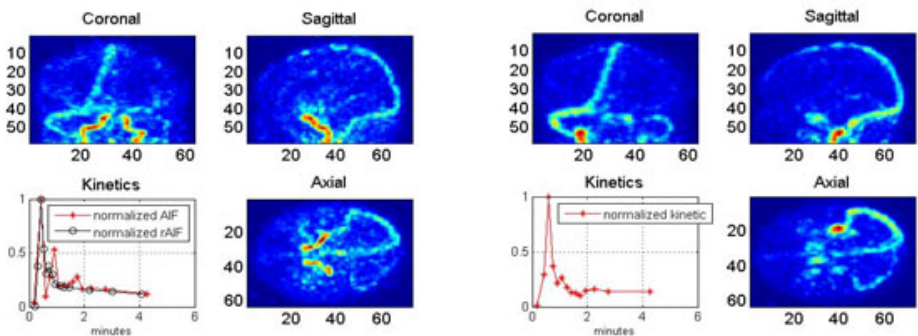

(a) Arterial

(b) Veinous
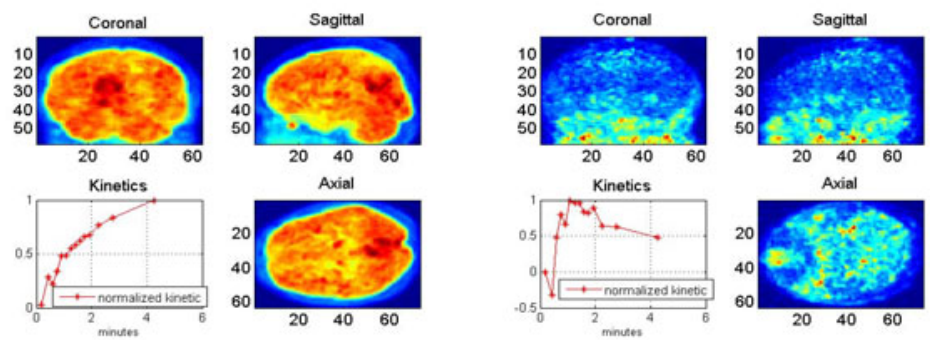

(c) Tissue
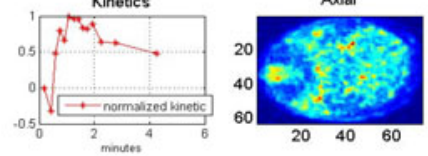

(d) Unidentified

Fig. 1. Estimated pharmacokinetics compartments 
distributions (estimated sources) according to the three views coronal (upper left), sagittal (upper right) and axial (lower right). Three of the estimated compartments are identified to be the Arterial compartment (Fig 1.a), the Veinous one (Fig 1.b) and the Tissue one (Fig 1.c). Fig 1.a (lower left) shows that the normalized estimated AIF correctly approximates the normalized rAIF obtained by blood sampling, which was the main objective. However, one may note negative values on the kinetic of the unidentified compartment (lower left of Fig 1.d) which we attribute to measure noise.

\section{Conclusions an Future Works}

In this paper, we present a geometrical method for separating nonnegative sources. In overdetermined case, the proposed method first reduces the dimension of mixed data by performing the classical PCA and afterward runs the SCSAUNS algorithm on the reduced data. The SCSA-UNS algorithm, estimates the mixing matrix by fitting a minimum volume simplicial cone to the scatter plot of observations. Unlike other geometrical methods that require local dominance or full additivity of the sources, SCSA-UNS only requires the mixed data to fill enough the simplicial cone generated by the mixing matrix. Simulations on synthetic data have showned that the proposed method always performs a good separation in noiseless case and runs faster than other MV methods. The proposed method also gave very promising results on real Dynamic PET images. As future works, we will investigate how to avoid the freezing situations without having to compute unfreezing matrices and we will consider the noisy case.

\section{References}

1. Puntonet, C., Mansour, A., Jutten, C.: A Geometrical Algorithm for Blind Source Separation. In: Gretsi 1995, Juan-les-Pins, pp. 273-276 (September 1995)

2. Babaie-Zadeh, M., Mansour, A., Jutten, C., Marvasti, F.: A Geometric Approach for Separating Several Speech Signals. In: Puntonet, C.G., Prieto, A.G. (eds.) ICA 2004. LNCS, vol. 3195, pp. 798-806. Springer, Heidelberg (2004)

3. Lazar, C., Nuzillard, D., Nowé, A.: A New Geometrical BSS Approach for Non Negative Sources. In: Vigneron, V., Zarzoso, V., Moreau, E., Gribonval, R., Vincent, E. (eds.) LVA/ICA 2010. LNCS, vol. 6365, pp. 530-537. Springer, Heidelberg (2010)

4. Chan, T.H., Ma, W.K., Chi, C.Y., Wang, Y.: A Convex Analysis Framework for Blind Separation of Non-Negative Sources. IEEE Transactions on Signal Processing 56, 5120-5134 (2008)

5. Li, J., Bioucas-Dias, J.M.: Minimum Volume Simplex Analysis: A Fast Algorithm to Unmix Hypersectral Data. In: IEEE International Symposium on Geoscience and Remote Sensing, vol. 3, pp. 250-253 (2008)

6. Chan, T.H., Chi, C.Y., Huang, Y.M., Ma, W.K.: A Convex Analysis-Based Minimum-Volume Enclosing Simplex Algorithm for Hyperspectral Unmixing. IEEE Transactions on Signal Processing 57, 4418-4432 (2009)

7. Craig, M.D.: Minimum-Volume Transforms for Remotely Sensed Data. IEEE Transactions on Geoscience and Remote Sensing 32, 542-552 (1994) 
8. Henry, R.C.: History and fundamentals of multivariate air quality receptor models. Chemometrics and Intelligent Laboratory Systems 37, 37-42 (1997)

9. Ouedraogo, W.S.B., Souloumiac, A., Jaidane, M., Jutten, C.: Simplicial Cone Shrinking Algorithm for Unmixing Nonnegative Sources. In: Accepted to ICASSP 2012 (2012)

\section{Appendix 1: Computing $\boldsymbol{R}_{k}^{l}$}

$W \geq 0$ and $Y=W^{-1} X \geq 0$ are the current estimations of $A$ and $S$ at iteration $k-1$ of sweep $l$, respectively. At iteration $k$, we look for a matrix $R_{k}^{l}$, so that $U=W R_{k}^{l}$ verify the following three conditions:

i) $U \geq 0$

ii) $V(U) \leq V(W)$

iii) $Z=\bar{U}^{-1} X \geq 0\left(Z=U^{-1} X=\left(R_{k}^{l}\right)^{-1} W^{-1} X=\left(R_{k}^{l}\right)^{-1} Y\right)$

The first two conditions are automatically satisfied because $W$ and $R_{k}^{l}$ are nonnegative and due to Proposition 1. From the definition of $R_{k}^{l}$ given by (6), one may demonstrate that $[Z]_{i j}=[Y]_{i j}-r_{i k}^{l}[Y]_{k j}, \forall 1 \leq i \leq n, \forall 1 \leq j \leq p$. For fixed $i,[Z]_{i j} \geq 0 \Leftrightarrow r_{i k}^{l} \leq \frac{[Y]_{i j}}{[Y]_{k j}}, \forall 1 \leq j \leq p$.

A convenient $R_{k}^{l}$ matrix can then be computed by taking:

$$
r_{k k}^{l}=1 \text { and } r_{i k}^{l}=\min _{1 \leq j \leq p} \frac{[Y]_{i j}}{[Y]_{k j}},[Y]_{k j} \neq 0 \text {, for } i \neq k
$$

\section{Appendix 2: Computing the Unfreezing Matrix $Q_{l}$}

Before giving details of computation of the unfreezing matrix $Q_{l}$, lets explain how arises the freezing situation. Given $W \geq 0$ and $Y=W^{-1} X \geq 0$ the current estimated mixing matrix sources respectively and $U=W R_{k}^{l}$ :

$V(U)=V(W) \Leftrightarrow R_{k}^{l}=I_{n} \Longleftrightarrow r_{i k}^{l}=\delta_{i k}$. For $i \neq k$ and according to (12), $r_{i k}^{l}=0 \Longleftrightarrow \exists 1 \leq j \leq p$ so $[Y]_{i j}=0$ and $[Y]_{k j} \neq 0$.

The freezing arises if, at sweep $l$, the algorithm finds $R_{k}^{l}=I_{n}, \forall 1 \leq k \leq n$. This situation happens when there are at least one zero value on each row of the current estimated sources matrix (i.e. when $\forall 1 \leq i \leq n, \exists 1 \leq j \leq p,[Y]_{i j}=0$ ). To avoid this problem, we suggest applying an orthogonal linear transformation $Q_{l}$ to $Y$ (and $Q_{l}^{T}$ to $W$ ) to delete the zeros values of $Y$ without increasing $V(W)$. We then introduce the unfreezing matrix $Q_{l}$ so that $X=W Q_{l}^{T} Q_{l} Y=H T$. The current estimated mixing matrix and sources become $H=W Q_{l}^{T}$ and $T=Q_{l} Y$. We look for a matrix $Q_{l}$ so that $Q_{l}^{T} Q_{l}=I_{n}$ and $T>0$.

For findind such a $Q_{l}$ matrix, we define the criterion $J$ by:

$$
J(Q)=\sum_{i=1}^{n} \sum_{j=1}^{p} 1_{T_{i j}} \text { where } 1_{T_{i j}}=\left\{\begin{array}{l}
1 \text { if } T_{i j}=0 \\
0 \text { elsewhere }
\end{array}\right.
$$

The unfreezing matrix $Q_{l}$ can be computed by solving the following equation:

$$
Q_{l}=\underset{Q^{T} Q=I_{n}}{\arg \min } J(Q)
$$

We developed a regularized gradient algorithm for solving the optimization problem (14) 9] but this method is not described here due to lack of space. 